\title{
ON THE REGULARITY OF MANY-PARTICLE DYNAMICAL SYSTEMS PERTURBED BY WHITE NOISE ${ }^{1}$
}

\author{
ANATOLI V. SKOROKHOD \\ Michigan State University \\ Department of Statistics and Probability \\ East Lansing, MI 48824-1027 USA \\ and \\ Ukrainian Academy of Sciences \\ The Institute of Mathematics \\ Kiev, Ukraine
}

(Received May, 1996; Revised October, 1996)

\begin{abstract}
ABSTRAC'T
We consider a system of finite number of particles that are moving in $R^{d}$ under mutual interaction. It is assumed that the particles are subjected to some additional random forces which cause diffusion motion of the particles. The latter is described by a system of stochastic differential equations of the first order for noninertia particles and the second order for inertial particles. The coefficient of the system are unbounded because the interaction force tends to infinity if the distance between two particles tends to zero. The system is called regular if no particle can hit the other. We investigate conditions of regularity.

This article is dedicated to the memory of Roland L. Dobrushin.

Key words: Many-particle Systems, Inertia Particle, Noninertia Particle, Stochastic Differential Equations, Martingale, Stopping Time, Regularity, Irregularity.
\end{abstract}

AMS (MOS) subject classifications: $60 \mathrm{H} 10,34 \mathrm{~F} 05,60 \mathrm{G} 46$.

\section{Introduction}

It is impossible to survey all aspects of the theory of randomly perturbed dynamical systems. I would like to mention the article of N.M. Krylov and N.N. Bogolubov [6] and the work of I.I. Gikhman [1], where the notion of stochastic differential equation was introduced to prove a wellknown statement of Krylov and Bogolubov. Stochastic differential equations became the main tool for the investigation of randomly perturbed dynamical systems. A variety of the results of such kind are collected in the book of R. Khasminskii [5]. I also use the results from the monographs by I.I. Gikhman and A.V. Skorokhod [2] and A.V. Skorokhod [9].

In monograph [8], the system of randomly interacting particles was considered under the assumption that random perturbations are generated by some Poisson random measures. The coefficients of corresponding stochastic equations are supposed to be smooth.

\footnotetext{
${ }^{1}$ Supported in part by ONR Grant NOOO14-96-0306.
} 
At that time, I discussed some of the results of this monograph with Roland Dobrushin and he asked me whether all the particles of the system could stick together. Trying to answer his question, I accomplished this article.

The description of nonperturbed several particle mechanical systems can be found in the book of Watson [10].

We consider the system of $N$ particles that are moving in the space $R^{d}$ and mutually interacting. It is supposed that the interaction force of two particles depends only on the distance between them; this force is directed from one particle to another (in the case of attraction) or it has the opposite direction (in the case of repulsion). Besides, the particles are subjected to some random forces which cause additional diffusion motion of the particles.

Let $x_{1}(t), \ldots, x_{N}(t)$ be the positions of the particles at time $t$. We assume that $x_{1}(t), \ldots, x_{N}(t)$ are stochastic processes satisfying some system of stochastic differential equations. The form of the equations depends on some additional assumptions

\section{Noninertia particles}

In this case, forces and random perturbations are acting directly on particles; the velocities of particles are not defined. The system of the stochastic differential equations is of the form

$$
d x_{k}(t)=N^{-1} \sum_{i=1}^{N} F_{i k} d t+\sigma d w_{k}(t), \quad k=1, \ldots, N
$$

where $F_{i k}$ is the force that is caused by $i$ th particle at the $k$ th one, $\sigma>0$ is a parameter, and $\left\{w_{i}(t), i=1, \ldots, N\right\}$ is a system of independent Wiener processes in $R^{d}$. in $R^{d}$.

We assume that $E w_{i}(t)=0, E\left(w_{i}(t), z\right)^{2}=t(z, z)$, where $z \in R^{d},(\cdot, \cdot)$ is the scalar product

\section{Inertia particles}

In this case, particles have velocities. We denote the velocity of the $i$ th particle by $\dot{x}_{i}(t)$. The system of stochastic differential equations is of the form

$$
d \dot{x}_{k}(t)=N^{-1} \sum_{i=1}^{N} F_{i k} d t+\sigma d w_{k}(t), \quad k=1, \ldots, N .
$$

\section{The assumptions on $\boldsymbol{F}_{i k}$}

(F1) We assume that there exists a function $F:(0, \infty) \rightarrow R$ that has continuous derivative and

$$
F_{i k}=F^{\prime}\left(\left|x_{i}(t)-x_{k}(t)\right|\right) \frac{x_{i}(t)-x_{k}(t)}{\left|x_{i}(t)-x_{k}(t)\right|} .
$$

It is supposed that the function $F$ satisfies one of the following conditions:

$(\boldsymbol{A F}) \quad F(u)>0, \quad F^{\prime}(u)<0, \quad F^{\prime \prime}(u)>0, u \in(0, \infty), \quad \lim _{u \rightarrow+\infty} F(u)>0$, and $\lim _{u \rightarrow 0} F^{\prime}(u)=$ $-\infty$.

$(\boldsymbol{R F})-F(u)$ satisfies condition $(\boldsymbol{A F})$.

In the case $(\boldsymbol{A F})$ particles are attracting and in the case $(\boldsymbol{R F})$ they are repulsing.

Because of conditions $(\boldsymbol{A F})$ and $(\boldsymbol{R F})$ and formula (3) the coefficients of equations (1) and (2) are unbounded and undefined if $x_{k}(t)=x_{i}(t)$ for some $k, i, t$.

We can consider the solutions of equations (1) and (2) only on the interval $[0, \zeta)$, where $\zeta$ is a stopping time for which $\Delta(t)=\prod_{i \neq k}\left|x_{i}(t)-x_{k}(t)\right|>0$ for $t<\zeta$ and $\Delta(\zeta-)=0$ if $\zeta<\infty$. 
The existence and uniqueness of the solution for any initial condition such that $\Delta(0)>0$ is the consequence of general theorems on local existence and uniqueness of the solution for stochastic differential equations (see, for example [2], p. 506).

A system is called regular if $P\{\zeta<\infty\}=0$ for any initial condition. This article is devoted to the investigation of the conditions under which the system is regular.

\section{Noninertia Particles}

\section{Two-particle systems}

We consider equation (1) for $N=2$ with $F_{i k}$ given the formula (3). We can write the following equation for $x_{1}(t)-x_{2}(t)=z(t)$ :

$$
d z(t)=F^{\prime}(|z(t)|) \frac{z(t)}{|z(t)|} d t+\sqrt{2} \sigma d w(t)
$$

where $w(t)=\frac{w_{1}(t)-w_{2}(t)}{\sqrt{2}}$ is also a Wiener process distributed as $w_{1}(t)$. Set

$$
X(t)=(z(t), z(t)) .
$$

$X(t)$ is a real-valued stochastic process that satisfies the relation

$$
d X(t)=\left[2 F^{\prime}\left(X^{\frac{1}{2}}(t)\right) X^{\frac{1}{2}}(t)+\left(2 \sigma^{2} d\right)\right] d t+2 \sqrt{2} \sigma(z(t), d w(t)) .
$$

(We use Ito's formula.) Set $2 \sigma^{2} d=\rho$ (here $d$ is the dimension of the phase space $R^{d}$ ). Let

$$
\widehat{w}(t)=\int_{0}^{t} 1_{\{s<\zeta\}}|z(s)|^{-1}(z(s), d w(s))+\int_{0}^{t} 1_{\{s>\zeta\}}|z|^{-1}(z, d w(s)),
$$

where $\zeta$ is a stopping for which $z(t)>0$ if $t<\zeta$ and $z(\zeta-)=0$ if $\zeta<\infty, z \in R^{d}, z \neq 0$ is a fixed vector. $\widehat{w}(t)$ is a Wiener process and $X(t)$ satisfies the stochastic differential equation:

$$
\left.d X(t)=\left[2 F^{\prime}\left(X^{\frac{1}{2}}(t)\right) X^{\frac{1}{2}}(t)+\rho\right] d t+2 \sqrt{2} \sigma(X(t))^{\frac{1}{2}} d \widehat{w}(t)\right) .
$$

We consider the solution of this equation with a positive initial value $X(0), \zeta=\sup \{t: X(t)>0\}$.

Set

$$
H(u)=u^{-\frac{d}{2}} \exp \left\{-\frac{1}{\sigma^{2}} F\left(u^{\frac{1}{2}}\right)\right\} .
$$

Theorem 1: a) $P\{\zeta=+\infty\}=1$ for any initial condition if and only if

$$
\int_{0}^{1} H(u) d u+\int_{0}^{1} H(u) \int_{u}^{1} H^{-1}(v) d v d u=+\infty .
$$

b) Assume that $\int_{0}^{1} H(u) \int_{0}^{1} H^{-1}(v) d v d u<\infty$. Then

$$
\lim _{X_{0} \rightarrow 0+} P\left\{\zeta<+\infty / X(0)=X_{0}\right\}=1 .
$$

Proof: The generator of the diffusion process satisfying equation (6) is of the form

$$
L f(x)=2 F^{\prime}(\sqrt{x}) \sqrt{x} f^{\prime}(x)+8 \sigma^{2} x f^{\prime \prime}(x)
$$

on the interval $(0, \infty)$. It is easy to check that the function $\Phi(x)=\int_{x}^{1} H(u) d u$ satisfies the equa- 

tion $L \Phi(x)=0$ and the function $\Psi(x)=\int_{x}^{1} H(u) \int_{u}^{1} H^{-1}(v) d v d u$ satisfies the equation $L \Psi(x)=$
-1. 42).

Now the proof of the theorem is the consequence of the results of W. Feller [4] (see also [9], p.

Remarks: 1) The result of Theorem 1 holds true if $F^{\prime}(u)$ is bounded on any interval $[\delta, \infty)$, $\delta>0$ and $F^{\prime \prime}(u)$ is locally bounded on $(0, \infty)$.

2) If $F(u)<0$ and $d \geq 3$ then the system is regular because

$$
\int_{0}^{1} H(u) d u \geq \int_{0}^{1} u^{\frac{1-d}{2}} d u=+\infty .
$$

3) Let $F(u)=c u^{-\gamma}, \gamma>0, c>0$, then the system is regular.

4) Let $F(u)=c \log \frac{1}{u}, c>0$, then the system is regular if $\frac{c}{2 \sigma^{2}} \leq \frac{d}{2}-1$ and the system is irregular if $\frac{c}{2 \sigma^{2}}>\frac{d}{2}-1$.

\section{The system of many noninertia particles}

We use expression (3) for $F_{i k}$ and set $F_{i i}=0$. Then

$$
d\left(x_{i}(t)-x_{j}(t)\right)=N^{-1} \sum_{k}\left(F_{i k}-F_{j k}\right) d t+\sigma\left(d w_{i}(t)-d w_{j}(t)\right)
$$

and

Introduce the function

$$
\begin{gathered}
d\left(x_{i}(t)-x_{j}(t), x_{i}(t)-x_{j}(t)\right)=2 N^{-1} \sum_{k}\left(F_{i k}-F_{j k}, x_{i}(t)-x_{j}(t)\right) d t+\left(2 d \sigma^{2}\right) d t \\
+2 \sigma\left(x_{i}(t)-x_{j}(t), w_{i}(t)-w_{j}(t)\right) .
\end{gathered}
$$

$$
R(t)=\sum_{i, j}\left(x_{i}(t)-x_{j}(t), x_{i}(t)-x_{j}(t)\right)
$$

Then

$$
\begin{gathered}
d R(t)=2 N^{-1} \sum_{k, i, j}\left(F_{i k}-F_{j k}, x_{i}(t)-x_{j}(t)\right) d t+2 N(N-1) d \sigma^{2} d t \\
+\sum_{i, j} 2 \sigma\left(x_{i}(t)-x_{j}(t), w_{i}(t)-w_{j}(t)\right) .
\end{gathered}
$$

It is easy to see that for $z_{1}, \ldots, z_{N} \in R^{d}$ we have that

So,

$$
\sum_{i, j}\left(z_{i}-z_{j}, \sum_{k}\left(F_{i k}-F_{j k}\right)\right)=N \sum_{i, j}\left(z_{i}-z_{j}, F_{i j}\right)
$$

Note that the process

$$
\begin{gathered}
d R(t)=\left(2 \sum_{i, j} F^{\prime}\left(\left|x_{i}(t)-x_{j}(t)\right|\right)\left|x_{i}(t)-x_{j}(t)\right|+2 N(N-1) d \sigma^{2}\right) d t \\
+\sum_{i, j} 2 \sigma\left(x_{i}(t)-x_{j}(t), w_{i}(t)-w_{j}(t)\right) .
\end{gathered}
$$

$$
\widetilde{R}(t)=2 \sigma \sum_{i, j} \int_{0}^{t}\left(x_{i}(s)-x_{j}(s), d w_{i}(s)-d w_{j}(s)\right)
$$

is a martingale. After some calculations we can obtain the following expression for its square characteristic:

$$
\langle\widetilde{R}, \widetilde{R}\rangle_{t}=8 \sigma^{2} N \int_{0}^{t} R(s) d s .
$$


Therefore,

$$
\widetilde{w}(t)=\int_{0}^{t} \frac{1}{\sqrt{N}} R^{-\frac{1}{2}}(s) \sum_{i, j}\left(x_{i}(s)-x_{j}(s), d w_{i}(s)-d w_{j}(s)\right)
$$

is a Wiener process.

Theorem 2: a) Let $F$ satisfy condition $(\boldsymbol{A F})$ and let there exist the limit $\lim _{t \rightarrow 0}\left(-t F^{\prime}(t)\right)=c, c \geq 0$, (it is possible that $\left.c=+\infty\right)$, if $0<c<+\infty$, then $c+t F^{\prime}(t)$ is bounded on the interval $(0, \delta)$ for some $\delta>0$.

The system is regular if $\frac{c}{2 \sigma^{2}}>\frac{d}{2}-1$ and the system is irregular if $\frac{c}{2 \sigma^{2}}<\frac{d}{2}-1$.

b) Let $\boldsymbol{F}$ satisfy condition $(\boldsymbol{R F})$. Then the system is regular.

Proof: We will use the following statement: if the system is irregular for $N=N_{0}$ then it is irregular for $N>N_{0}$. To prove this statement we only have to note that for the system of $N_{0}+1$ particles we can choose the initial value $x_{N_{0}+1}(0)$ so far from $x_{0}(0), \ldots, x_{N_{0}}(0)$ that the influence of $x_{N_{0}+1}(t)$ on the $x_{0}(t), \ldots, x_{N_{0}}(t)$ is negligibly small.

So the system is irregular for all $N$ if it is irregular for $N=2$. But there exists the possibility that the system is regular for $N=2,3, \ldots, N_{0}$ and it is irregular for $N=N_{0}+1$. In this case

where

$$
P\{\widehat{\zeta}<\infty\}=1,
$$

$$
\widehat{\zeta}=\sup \left\{t: \sum_{i, j \leq N_{0}+1}\left|x_{i}(t)-x_{j}(t)\right|>0\right\}
$$

In part $a$ ) of the theorem we consider first $F^{\prime}(t)=-\frac{c}{t}$. Equation (9) can be rewritten in the form:

$$
d R(t)=\left(-2 c N(N-1)+2 N(N-1) d \cdot \sigma^{2}\right) d t+\sqrt{8 \sigma^{2} N} R^{\frac{1}{2}}(t) d \widetilde{w}(t)
$$

As it follows from Theorem 1, the solution of this equation is irregular if

$$
(N-1)\left[\frac{c}{2 \sigma^{2}}-\frac{d}{2}\right]>-1
$$

and the solution is regular if

It is easy to see that if

$$
(N-1)\left[\frac{c}{2 \sigma^{2}}-\frac{d}{2}\right] \leq-1
$$

$$
\left(N_{0}-1\right)\left[\frac{c}{2 \sigma^{2}}-\frac{d}{2}\right] L \leq-1
$$

then $N_{0}\left[\frac{c}{2 \sigma^{2}}-\frac{d}{2}\right]<-1$.

Assume that $F(t)$ satisfies condition $a)$ with $c \in(0, \infty)$. Denote by $Y(t)$ the solution of stochastic differential equation:

$$
d Y(t)=\alpha d t+\sqrt{8 \sigma^{2} N} Y^{\frac{1}{2}}(t) d \widetilde{w}(t)
$$

and let $\zeta_{Y}=\sup \{t: Y(s)>0$ for $s \in(0, t)\}, \alpha=\left(-2 c+2 d \cdot \sigma^{2}\right) N(N-1)$.

Set

$$
\beta(t)=2 \sum_{i, j}\left[F^{\prime}\left(\left|x_{i}(t)-x_{j}(t)\right|\right)\left|x_{i}(t)-x_{j}(t)\right|+c\right] .
$$

It follows from condition a) that $\beta(t)=R^{\frac{1}{2}}(t) \gamma(t)$, where $\gamma(t)$ is a bounded measurable adapted function. It follows from relation (9) that 


$$
d R(t)=\alpha d t+\sqrt{8 \sigma^{2} N} R^{\frac{1}{2}}(t)\left[d \widetilde{w}(t)+\left(8 \sigma^{2} N\right)^{-\frac{1}{2}} \gamma(t) d t\right]
$$

Since measures corresponding to the stochastic processes $\widetilde{w}(t)$ and $\widetilde{w}(t)+\int_{0}^{t}\left(8 \sigma^{2} N\right)^{-\frac{1}{2}} \gamma(s) d s$ are equivalent due to Girsanov's theorem (see [3]), the measures corresponding to $Y(t)$ and $R(t)$ are equivalent if $Y(0)=R(0)$.

Consequently, $P\{\zeta<\infty\}>0$ if $P\left\{\zeta_{Y}<\infty\right\}>0$ and $P\{\zeta<\infty\}=0$ if $P\left\{\zeta_{Y}<\infty\right\}=0$. So, part $a)$ is proved for $c \in(0, \infty)$. We also have to consider the case $c=0$ and the case $c=+\infty$. For this, we need the following statement.

Lemma 1: Let $F$ and $F^{*}$ satisfy the conditions of part a) of the theorem. We denote by $S$ and $S^{*}$ the corresponding systems of stochastic differential equations. Let $F^{\prime}(u) u \leq F^{* \prime}(u) u$. Then, if $S^{*}$ is irregular then $S$ is also irregular; hence if $S$ is regular, then $S^{*}$ is regular.

The proof of the statement can be rendered in the same way as the comparison theorem (see [7], p. 124).

To prove statement $b$ ) we note that Lemma 1 is true if $F$ and $F^{*}$ satisfy condition ( $R \boldsymbol{F}$ ). But if $F=0$ then the equation for $R$ is of the form:

$$
d R(t)=2 \cdot \sigma^{2} N(N-1) d t+\sqrt{8 \sigma^{2} N} \sqrt{R(t)} d \widetilde{w}(t) .
$$
The function $H(u)$ for this equation is $H(u)=u^{-\frac{d}{2}(N-1)}$ and $\int_{0}^{1} H(u) d u=+\infty$ if $N>2$ for
any $d=1,2 \ldots$.

So the solution of equation (13) is regular. Therefore, it is regular for any $F$ satisfying condition $(\boldsymbol{R F})$.

\section{Inertia Particles}

\section{Two-particle system}

We consider equation (2) for $N=2$ with $F_{i k}$ given by formula (3). Set $z(t)=x_{1}(t)-x_{2}(t)$. Then $z(t)$ is a solution of the equation

where $w(t)$ is the same as in equation (4).

$$
d \dot{z}(t)=F^{\prime}(|z(t)|) \frac{z(t)}{|z(t)|} d t+\sqrt{2} \sigma d w(t)
$$

\section{Results for unperturbed systems}

Let $\sigma=0$. We introduce two functions:

and

$$
H(t)=\frac{1}{2}(\dot{z}(t), \dot{z}(t))-F(|z(t)|)
$$

$$
M(t)=(z(t), z(t))(\dot{z}(t), \dot{z}(t))-(z(t), \dot{z}(t))^{2} .
$$

Hence, $H(t)=H_{0}, M(t)=M_{0}$, and $H_{0}, M_{0}$ are determined by the initial values $z(0), \dot{z}(0)$. It follows from (15) and (16) that

$$
(z(t), \dot{z}(t))^{2}=(z(t), z(t))\left[2 H_{0}+2 F(|z(t)|)\right]-M_{0}
$$

Note that $M_{0} \geq 0$.

We consider the conditions under which there exist $z(0)$ and $\dot{z}(0)$ such that $z\left(t_{0}\right)=0$ for some $t_{0}>0$. In this case we will say that the system is irregular. If $z(t) \neq 0$ for any initial condition and $t>0$ then we say that the system is regular. 
I. Let $F$ satisfy condition $(\boldsymbol{R F})$. Then the system is regular. This follows from the relation:

$$
-F(|z(t)|)<H(t)=H_{0} .
$$

So $|z(t)|>\lambda$ if $-F(\lambda)=H_{0}$.

II. Let $\boldsymbol{F}$ satisfy condition $(\boldsymbol{A F})$. Then

(i) if $\lim _{u \rightarrow 0} u^{2} F(u)>0$ the system is irregular;

(ii) if $\lim _{u \rightarrow 0} u^{2} F(u)=0$ the system is irregular but for any initial condition for which $M_{0}>0, z(t)$ satisfies the relation: $|z(t)|>0$ for all $t>0$.

\section{Perturbed two-particle system}

We consider equation (14) for $\sigma>0$. In this case the functions $H(t)$ and $M(t)$ are not constant. We can obtain the following stochastic differential equations for these functions:

$$
d H(t)=\rho d t+\sqrt{2 H(t)+2 F(|z(t)|)} d \widetilde{w}(t)
$$

where $\rho=2 d \sigma^{2}$ and

$$
\widetilde{w}(t)=\int_{0}^{t}|\dot{z}(s)|^{-1}(\dot{z}(s), d w(s))
$$

is a Wiener process. We use the relation $|\dot{z}(t)|^{2}=2 H(t)+2 F(z(t))$

$$
d M(t)=\left(\rho-2 \sigma^{2}\right)(z(t), z(t)) d t+\sqrt{8} \sigma[(z(t), z(t)) M(t)]^{\frac{1}{2}} d \widehat{w}(t),
$$

where

$$
\begin{gathered}
\widehat{w}(t)=\int_{0}^{t}[(z(s), z(s)) M(s)]^{-\frac{1}{2}}(z(s), z(s))(\dot{z}(s), d w(s)) \\
-(\dot{z}(s), z(s))(z(s), d w(s))
\end{gathered}
$$

is also a Wiener process.

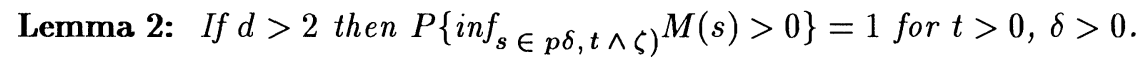

Proof: Let $\tau_{u}$ be a solution of the equation

It is easy to check that

$$
u=\int_{0}^{\tau}(z(s), z(s)) d s .
$$

$$
w^{*}(u)=\int_{0}^{\tau}|z(s)| d \widehat{w}(s)
$$
is a Wiener process on the interval $\left[0, \int_{0}^{\zeta}(z(s), z(s)) d s\right)$ and $M\left(\tau_{u}\right)$ satisfies the stochastic differen-
tial equation

Since

$$
d M\left(\tau_{u}\right)=\left(\rho-2 \sigma^{2}\right) d u+\sqrt{8} \sigma \sqrt{M\left(\tau_{u}\right)} d w^{*}(u) .
$$

$$
2 \frac{\rho-2 \sigma^{2}}{8 \sigma^{2}}=\frac{d-1}{2}
$$

the solution of the equation

is positive for $t>0$.

$$
d M=\left(\rho-2 \sigma^{2}\right) d u+\sqrt{8} \sigma \sqrt{M} d w^{*}(u)
$$

Lemma 3: Let $\alpha>\frac{1}{2}$. Then for any $\epsilon>0$ there exists a constant $c_{\alpha}(\epsilon)$ for which

$$
P\left\{\sup _{t>0} \frac{|\mu(t)|}{1+\langle\mu, \mu\rangle_{t}^{\alpha}}>c_{\alpha}(\epsilon)\right\}<\alpha
$$


for any continuous real-valued martingale $\mu(t)$ with the square characteristic $\langle\mu, \mu\rangle_{t}$.

To prove the statement we note that for the Wiener process $w(t)$ we have

$$
P\left\{\sup _{t>0} \frac{|w(t)|}{1+|t|^{\alpha}}<\infty\right\}=1 \text {. }
$$

Lemma 4: Let $\boldsymbol{F}$ satisfy condition $(\boldsymbol{A F})$. Then for any $\alpha \in\left(\frac{1}{2}, 1\right)$ and $t>0$,

$$
P\left\{\sup _{s<t \wedge \zeta} \frac{|H(s)|}{F^{\alpha}(|z(s)|)}<\infty\right\}=1 .
$$

Proof: Denote $\tilde{H}(t)=H(t)-\rho t$. Then,

$$
\begin{gathered}
\langle\widetilde{H}, \tilde{H}\rangle_{t}=\int_{0}^{t}[2 H(s)+2 F(|z(s)|)] d s \\
\leq \int_{0}^{t}[2|H(s)|+4 F(|z(s)|)] d s \leq \rho t^{2}+\int_{0}^{t} 2|\tilde{H}(s)| d s+\int_{0}^{t} 2 F(|z(s)|) d s .
\end{gathered}
$$

It follows from Lemma 3 that for all $s<t \wedge \zeta$,

$$
|\widetilde{H}(s)| \leq\left(1+\langle\widetilde{H}, \widetilde{H}\rangle_{s}^{\alpha}\right) \theta
$$

where $\theta$ is a random variable. Using this relation we can accomplish the proof.

Remark: Let $F$ satisfy condition $(\boldsymbol{R F})$. Then,

$$
\langle\widetilde{H}, \widetilde{H}\rangle_{t} \leq \rho t^{2}+\int_{0}^{t} 2|\widetilde{H}(s)| d s .
$$

Using Lemma 3 we can prove that for any $t>0$,

$$
P\left\{\sup _{s<t \wedge \zeta}|H(s)|<\infty\right\}=1
$$

Theorem 3: 1) Let $\boldsymbol{F}$ satisfy condition $(\boldsymbol{A F})$. Then the system is irregular if

a) $\quad \lim _{u \rightarrow 0} u^{2} F(u)=+\infty$,

b) $\quad \lim _{u \rightarrow 0} u^{2} F(u)=c>0$.

The system is regular if

c) $\quad \lim _{u \rightarrow 0} u^{2} F(u)=0, d>2$.

2) Let $F$ satisfy condition $(\boldsymbol{R F})$. Then the system is regular.

Proof: 1) For perturbed systems the following relation holds:

$$
(z(t), \dot{z}(t))^{2}=(z(t), z(t))[2 H(t)+4 F(|z(t)|)]-m(t) .
$$

Suppose that $(z(0), \dot{z}(0))<0$, and

$$
(z(t), z(t))[2 H(t)+4 F(|z(t)|)]-M(t)>\nu^{2}, \quad \nu>0,
$$

for $t \in[0, \delta \wedge \zeta)$. Then $(z(t), \dot{z}(t))<0$ on the interval $[0, \delta \wedge \zeta)$. Since

$$
(z(t), \dot{z}(t))=\frac{1}{2} \frac{d}{d t}|z(t)|^{2},
$$

$|z(t)|$ decreases and $F(|z(t)|)$ increases on the interval $[0, \zeta \wedge \delta)$. If $\zeta>\delta$, then

$$
(z(\delta), z(\delta))<(z(0), z(0))-\frac{1}{2} \nu \delta
$$


but this is impossible if $(z(0), z(0))$ is small enough.

Let $\dot{z}(0)=-\lambda z(0), \lambda>0$. Then $M(0)=0$. It follows from equation (18) that there exists such $\delta>0$ that

$$
P\left\{\sup _{s<t \wedge \zeta} M(t)>\epsilon\right\}<\epsilon \text { if }|z(t)| \leq 1 \text { for } t \in[0, \delta \wedge \zeta)
$$

Using Lemma 4 we can find $|x(0)|, \nu$, and $\lambda$ for which $(21)$ is true and $(z(0), z(0))-\frac{1}{2} \nu \delta<0$. Thus statements $a$ ) and $b$ ) are proved.

To prove statement $1 c$ ) note that for $\zeta<\infty$, we have

$$
\lim _{t \uparrow \zeta}(z(t), z(t))[2 H(t)+4 F(|z(t)|)]=0 .
$$

But Lemma 2 implies that $M(\zeta-)>0$, so

Therefore $\zeta=+\infty$ with probability 1 .

$$
\lim _{t \uparrow \zeta}(z(t), \dot{z}(t))^{2}<0
$$

2) Note that $|F(|z(t)|)| \leq H(t)$. The proof of statement 2) is the consequence of the remark.

\section{The system of many inertia particles}

The corresponding system of stochastic differential equations is of the form

$$
d \dot{x}_{i}(t)=N^{-1} \sum_{k=1}^{N} F^{\prime}\left(\left|x_{i}(t)-x_{k}(t)\right|\right) \frac{x_{i}(t)-x_{k}(t)}{\left|x_{i}(t)-x_{k}(t)\right|}+\sigma d w_{i}(t) .
$$

Set

$$
H(t)=\sum_{i \neq j}\left(\frac{1}{2}\left|\dot{x}_{i}(t)-\dot{x}_{j}(t)\right|^{2}-F\left(\left|x_{i}(t)-x_{j}(t)\right|\right)\right) .
$$

Then,

$$
\begin{gathered}
d H(t)=\sum_{i, j}\left[\left(\dot{x}_{i}(t)-\dot{x}_{j}(t), N^{-1} \sum_{k} F_{i k}-F_{j k}\right)-F^{\prime}\left(\left|x_{i}(t)-x_{j}(t)\right|\right)\right. \\
\left.\times \frac{\left(x_{i}(t)-x_{j}(t), \dot{x}_{i}(t)-\dot{x}_{j}(t)\right)}{\left.\mid x_{i}(t)-x_{j}(t)\right\}}\right] d t+d \cdot \sigma^{2} N(N-1) d t \\
+\sigma \sum_{i \neq j}\left(\dot{x}_{i}(t)-\dot{x}_{j}(t), d w_{i}(t)-d w_{j}(t)\right) .
\end{gathered}
$$

We introduce the real-valued Wiener process

$$
\widetilde{w}(t)=\frac{1}{\sqrt{N}} \int_{0}^{t}\left(\sum_{i, j}\left|\dot{x}_{i}(s)-\dot{x}_{j}(s)\right|^{2}\right)^{-\frac{1}{2}} \sum_{i, j}\left(\dot{x}_{i}(s)-\dot{x}_{j}(s), d w_{i}(s)-d w_{j}(s)\right) .
$$

Note that the first sum in the right-hand side of equation (23) equals zero due to relation (8).

Set

$$
G(t)=\sum_{i \neq j} 2 F\left(\left|x_{i}(t)-x_{j}(t)\right|\right)
$$

Then,

$$
\sum_{i \neq j}\left|\dot{x}_{j}(t)-\dot{x}_{j}(t)\right|^{2}=2 H(t)+G(t)
$$

Therefore, $H(t)$ satisfies the stochastic differential equation

$$
d H(t)=d \cdot \sigma^{2} N(N-1) d t+\sigma \sqrt{2 H(t)+G(t)} \sqrt{N} d \widetilde{w}(t) .
$$

Let $R(t)$ be determined by relation (7). Then, 
and

$$
\dot{R}(t)=2 \sum_{i \neq j}\left(x_{i}(t)-x_{j}(t), \dot{x}_{j}(t)-\dot{x}_{j}(t)\right)
$$

$$
\begin{gathered}
d \dot{R}(t)=2 \sum_{i, j}\left|\dot{x}_{i}(t)-\dot{x}_{j}(t)\right|^{2} d t \\
+2 \sum_{i, j}\left(x_{i}(t)-x_{j}(t), N^{-1} \sum_{k} F_{i k}-F_{j k}\right) d t+2 \sigma \sum_{i, j}\left(x_{i}(t)-x_{j}(t), d w_{i}(t)-d w_{j}(t)\right) \\
=\left(2 H(t)+2 \sum_{i, j} \Phi\left(\left|x_{i}(t)-x_{j}(t)\right|\right)\right) d t+\sqrt{8 \sigma^{2} N R(t)} d \widetilde{w}(t),
\end{gathered}
$$

where

$$
\Phi(u)=2 F(u)+u F^{\prime}(u)=\frac{\left(u^{2} F(u)\right)^{\prime}}{u}
$$

and $\widetilde{w}(t)$ is the Wiener process determined by formula (10). Note that in equalities (27) we used relation (8).

Theorem 4: 1) Assume $F$ satisfies condition $(\boldsymbol{A F})$. If

(i) there exists the limit $\lim _{u \rightarrow 0} u^{-2} F(u) \in(0,+\infty]$, then the system is irregular;

(ii) $\lim _{u \rightarrow 0} u^{-2} F(u)=0$ and additionally the function $\Phi$ satisfies the following conditions:

$(\alpha) \lim _{u \rightarrow 0}(\Phi(u) / F(u))>0$,

( $\beta) \quad \Phi(\sqrt{u})$ is a convex up function,

then the system is regular.

2) If $\boldsymbol{F}$ satisfies condition ( $\boldsymbol{R F}$ ) then the system is regular.

Proof: The system is irregular in case $1(i)$ because it is irregular for $N=2$.

Consider case $1(i i)$. We can rewrite relation (27) in the form

$$
d \dot{R}(t)=\lambda N(N-1) \Phi\left(\sqrt{\frac{1}{N(N-1)} R(t)}\right) d t+V(t) d t+\sqrt{8 \sigma^{2} N R(t)} d \widetilde{w}(t),
$$

where $V(t)=V_{1}(t)+V_{2}(t), 0<\lambda<2$, and

$V_{1}(t) \geq 0$ because of $(\beta)$,

$$
V_{1}(t)=2 \sum_{i, j} \Phi\left(\left|x_{i}(t)-x_{j}(t)\right|\right)-2 N(N-1) \Phi\left(\sqrt{\frac{1}{N(N-1)} R(t)}\right) .
$$

$$
V_{2}(t)=(4-\lambda) N(N-1) \Phi\left(\sqrt{\frac{1}{N(N-1)} R(t)}\right)+4 H(t) .
$$

Because of Lemma $4, V_{2}(t)$ is positive if $R(t)$ is small enough. So we can compare $R(t)$ with the solution of the equation

$$
d \dot{R}^{*}(t)=\lambda N(N-1) \Phi\left(\sqrt{\frac{1}{N(N-1)} R^{*}(t)}\right) d t++\sqrt{8 \sigma^{2} N R^{*}(t)} d \widetilde{w}(t) .
$$

Note that this is an equation for $\left|x_{1}(t)-x_{2}(t)\right|^{2}$.

It follows from Theorem 3 that $P\left\{R^{*}(t)>0, t \in[0, \infty)\right\}=1$. Since $R(t)>R^{*}(t)$ in a neighborhood of $\zeta, \zeta=+\infty$ with probability 1 .

The proof of part 2) is the same as in Theorem 3.

\section{References}

[1] Gikhman, I.I., On the theory of differential equations of random processes I, II, Ukrani. 
Math. Zh. 2:4 (1950), 37-63, 3 (1951), 317-339; English trans. in Amer. Math. Soc. Transl. 1:2 (1955).

[2] Gikhman, I.I. and Skorokhod, A.V., Stochastic Differential Equations and Their Applications, "Naukova Dumka", Kiev 1982 (Russian).

[3] Girsanov, I.V., On the transformation of a certain class of stochastic processes by an absolutely continuous substitution of a measure, Theory of Prob. and Its Applications 5 (1960), 285-301.

[4] Feller, W., Diffusion processes in one dimension, Trans. Amer. Math. Soc. 77 (1954), 1-31.

[5] Khasminskii, R.Z., Stochastic Stability of Differential Equations, Sijthoff and Noordoff, Alphen aan den Rijn 1980.

[6] Krylov, N.M. and Bogolubov, N.N. Surles équations de Fokker-Planck déduites dans la théorie de perturbations à l'aide d'une méthode basée sur les properiétés spectrales de l'hamiltonien perturbateur, Zap. Kafedr. Mat. Fiz. Inst. Budivel. Mat. Akad. Nauk Ukrain. SSR 4 (1939), 81-157.

[7] Skorokhod, A.V., Studies in the Theory of Random Processes, Addison-Wesley, Reading, MA 1965.

[8] Skorokhod, A.V., Stochastic Equations for Complex Systems, Reidel 1988.

[9] Skorokhod, A.V., Asymptotic Methods in the Theory of Stochastic Differential Equations, Am. Math. Soc. Trans. of Math. Monographs 1989.

[10] Watson, K.M., Topics in Several Particle Dynamics, Holden-Day, San Francisco 1967. 


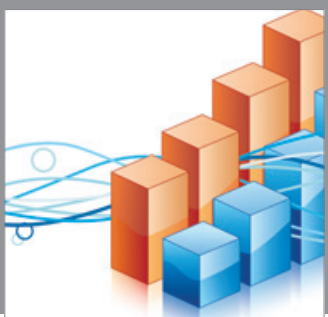

Advances in

Operations Research

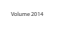

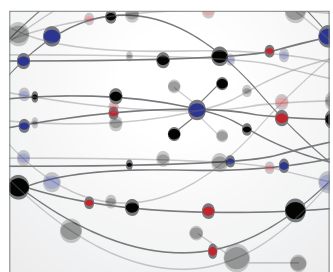

\section{The Scientific} World Journal
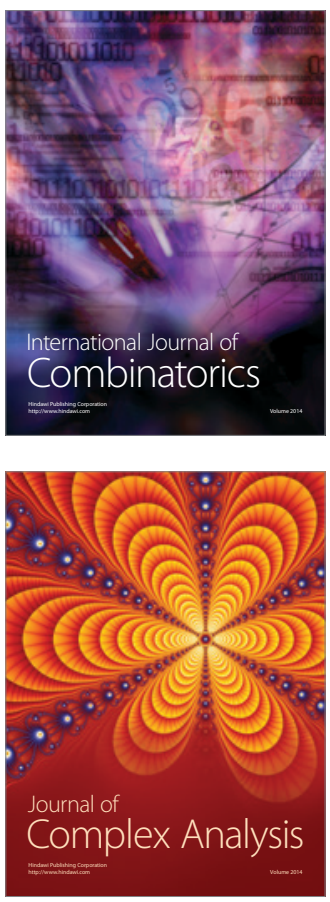

International Journal of

Mathematics and

Mathematical

Sciences
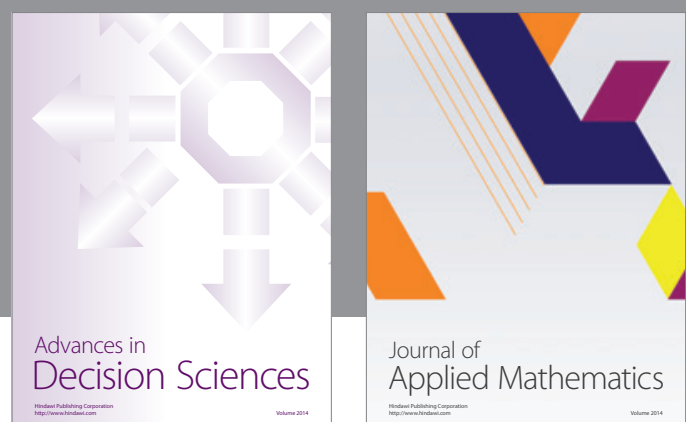

Journal of

Applied Mathematics
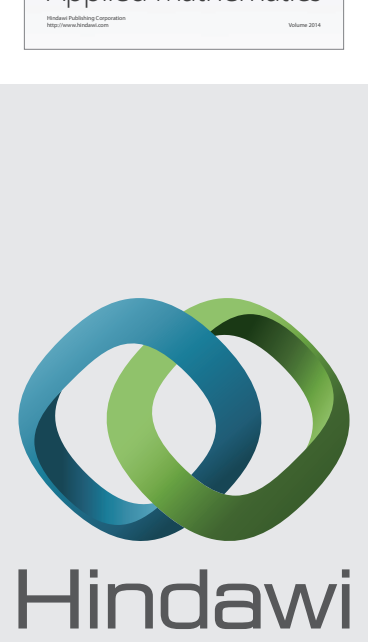

Submit your manuscripts at http://www.hindawi.com
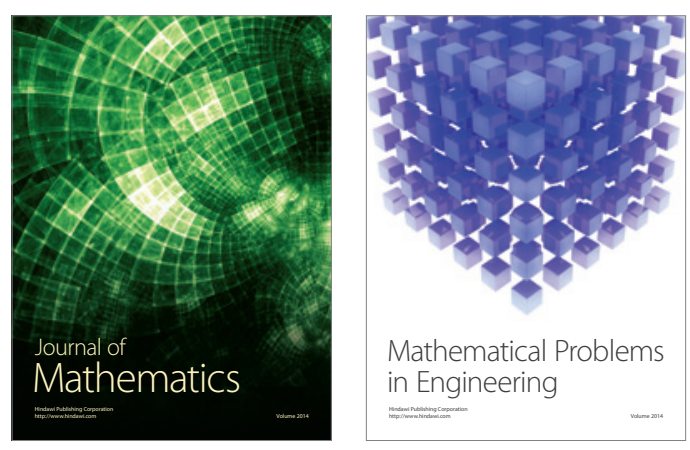

Mathematical Problems in Engineering
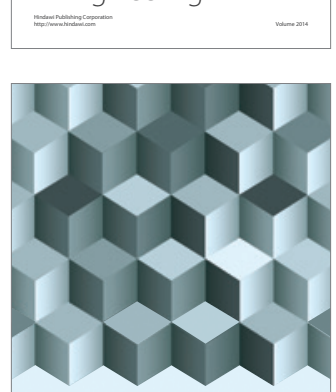

Journal of

Function Spaces
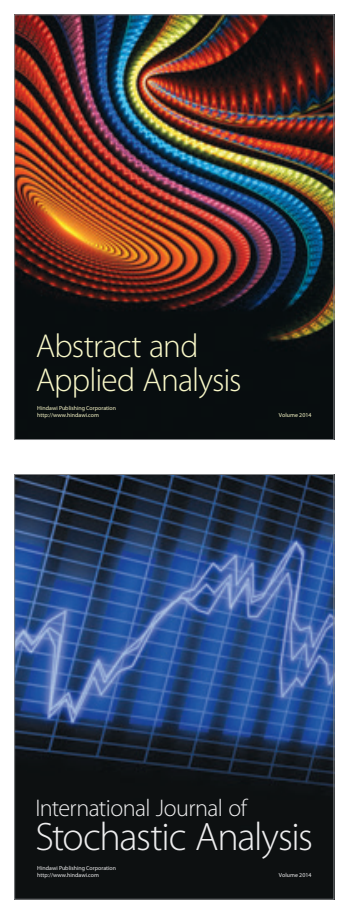

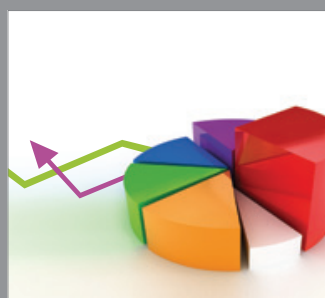

ournal of

Probability and Statistics

Promensencen
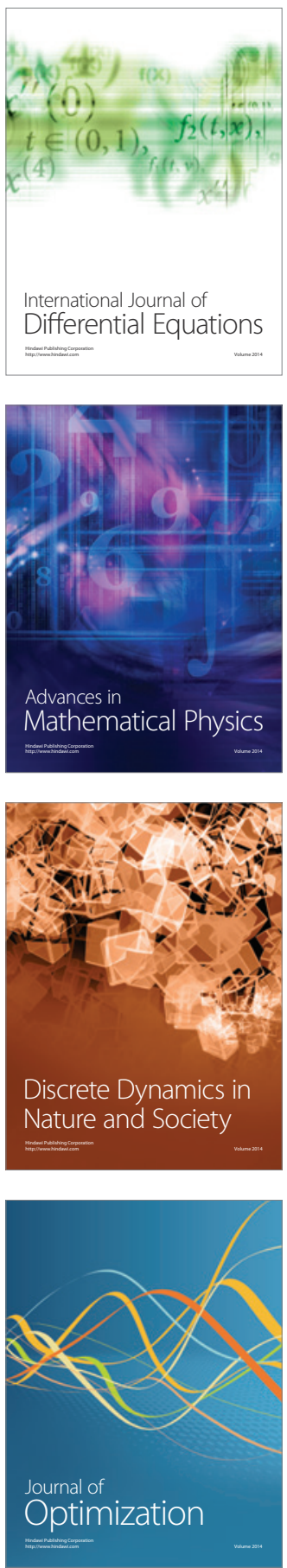